\title{
Coherence Properties of Coupled Chaotic Map Lattices
}

\author{
M. JANOWICZ ${ }^{a, b, *}$ AND A. OREOWSKI ${ }^{a, b}$ \\ ${ }^{a}$ Chair of Computer Science, Warsaw University of Life Sciences, Nowoursynowska 159, 02-766 Warsaw, Poland \\ ${ }^{b}$ Institute of Physics, Polish Academy of Sciences, al. Lotników 32/46, 02-668 Warsaw, Poland \\ Strong global correlations in the systems of coupled chaotic map lattices based on a modified logistic map are \\ investigated. It is shown that, in the parameter range close to the edge of chaos as defined for an individual map, \\ the systems exhibit off-diagonal long-range order and single-particle reduced density matrices defined in a natural \\ way possess one strongly dominant eigenvalue. In addition, pattern formation [13] in the above systems has been \\ investigated.
}

PACS: 05.45.Ra, 45.70.Qj, 03.75.Hh, 03.75.Nt

\section{Introduction}

Coupled map lattices (CMLs) [1, 2], that is systems of coupled maps which simulate spatially extended non-linear systems, have long become a useful tool to investigate spatiotemporal chaos and other non-linear phenomena [3-6]. Several CMLs have found interesting applications in physical modeling. One should mention here CMLs developed to describe the Rayleigh-Benard convection [7], dynamics of boiling [8,9], formation and dynamics of clouds [10], crystal growth processes and hydrodynamics of two-dimensional flows [11].

In our previous work [12] we have argued that CMLs based on the ubiquitous logistic map exhibit properties which are characteristic for the Bose-Einstein condensate. We have done this by describing CMLs with the help of a variable interpreted as a classical field defined on discrete space-time. This has allowed us to define the single-particle reduced density matrix in a natural way. That latter quantity enables one to give precise quantitative meaning to the terms "correlations", "coherence" and "long-range order" which are often loosely attributed to the spatially extended classical systems. It has turned out that CMLs based on the logistic map exhibit, for a broad range of parameters, off-diagonal long-range order. What is more, there exists one dominant eigenvalue of the reduced density matrix as well as a single dominant mode in the Fourier transform of the field describing CML. It is to be noted that the CMLs cannot of course be called "condensates", first of all because they are merely somewhat remote models of reality, and not physical systems. In addition, in those models there are no natural first integrals like the energy or the number of particles. Therefore, we say that CMLs exhibit "condensate-like" behavior.

Needless to say, the subject requires further investigations. One natural path to follow is checking for

\footnotetext{
* corresponding author; e-mail: mjanow@ifpan.edu.pl
}

the condensate-like behavior (or, more generally, strong global correlations and coherence) of some other, similar systems. It is the purpose of this work to present some results for CMLs based on modified logistic map such that every individual map can take negative values. In addition, we provide some results concerning pattern formation in the above CMLs for we believe that the subject is very far from being exhausted in spite of the existence of already classic papers on the subject by Kapral and Kaneko [5, 6, 11].

Our analysis is in the spirit of classical field theory, especially the Gross-Pitaevskii equation which is very extensively used in the theory of Bose-Einstein condensation $[14,15]$. Applications of the classical field-theoretical methods in the physics of condensates have been described, e.g., in [16-18].

The main body of this work is organized as follows. The mathematical model as well as the basic definitions of reduced density matrix and reduced wave function are introduced in Sect. 2. Section 3 provides a justification of our claim that the coupled map lattices based on modified logistic map exhibit properties which are analogous to those of the Bose-Einstein condensates (BEC). The description of numerical results concerning pattern formation are contained in Sect. 4, while Sect. 5 comprises a few concluding remarks.

\section{The model}

We consider a classical field $\psi(x, y, t)$ defined on a two-dimensional spatial lattice. Its evolution in (dimensionless, discrete) time $t$ is given by the following equation:

$$
\begin{aligned}
& \psi(x, y, t+1)=(1-4 d) f(\psi(x, y, t)) \\
& \quad+d[f(\psi(x+1, y, t))+f(\psi(x-1, y, t)) \\
& \quad+f(\psi(x, y+1, t))+f(\psi(x, y-1, t))],
\end{aligned}
$$

where the function $f$ is given by 


$$
f(\psi)=1-a \psi^{2},
$$

and the parameters $a$ and $d$ are constant. The set of values taken by $\psi$ is the interval $[-1,1]$, so that negative values of $\psi$ are allowed unlike in the work [12].

A single map of the form $\psi(t+1)=f(\psi(t))$ exhibits the accumulation of period-doubling at $a=1.40155 \ldots$ and the band merging from period-2 band to a single band state at $a=1.542 \ldots$

In the following the coefficient $a$ will be called the "non-linear parameter" while the coefficient $d$ will be called the "diffusion constant". It is assumed that $\psi$ satisfies the periodic boundary conditions on the borders of simulation box. The size of that box is $N \times N$. All our simulations have been performed with $N=256$.

Let $\tilde{\psi}$ be the two-dimensional discrete Fourier transform of $\psi$,

$$
\tilde{\psi}(m, n)=\sum_{x=0}^{N-1} \sum_{y=0}^{N-1} \mathrm{e}^{2 \pi \mathrm{i} m x / N} \mathrm{e}^{2 \pi \mathrm{i} n y / N} \psi(x, y) .
$$

Thus, $\tilde{\psi}$ may be interpreted as the momentum representation of the field $\psi$.

Below we investigate the relation between a CML described by Eq. (1) and a Bose-Einstein condensate. Therefore, let us invoke the basic characteristics of the latter which are so important that they actually form a part of its modern definition. These are [15, 19, 20]:

1. The presence of one eigenvalue of the one-particle reduced density matrix which is much larger than all other eigenvalues.

2. The presence of off-diagonal long-range order (ODLRO).

The property (1) corresponds to the well-known intuitive definition of the Bose-Einstein condensate. Taking into account that the following decomposition of the one-particle reduced density matrix $\rho^{(1)}$ has the following decomposition eigenvalues $\lambda_{j}$ and eigenvectors $\left|\phi_{j}\right\rangle$ :

$$
\rho^{(1)}=\sum_{j} \lambda_{j}\left|\phi_{j}\right\rangle\left\langle\phi_{j}\right|
$$

we can realize that if one of the eigenvalues is much larger than the rest, then the majority or at least a substantial fraction of particles is in the same single-particle quantum state.

In addition, for an idealized system of the Bose particles with periodic boundary conditions and without external potential, the following signature of condensation is also to be noticed:

3. The population of the zero-momentum mode is much larger than population of all other modes.

The properties (1) and (2) acquire quantitative meaning only if the one-particle reduced density matrix is defined. Since our model is purely classical, the definition of that density matrix is not obvious. We can use, however, the classical-field approach to the theory of BoseEinstein condensation $[17,21]$ and define the quantities:

$$
\bar{\rho}\left(x, x^{\prime}\right)=\left\langle\sum_{y=0}^{N-1} \psi(x, y) \psi\left(x^{\prime}, y\right)\right\rangle_{t},
$$

and

$$
\rho\left(x, x^{\prime}\right)=\bar{\rho}\left(x, x^{\prime}\right) / \sum_{x} \bar{\rho}(x, x) .
$$

We shall call the quantity $\rho\left(x, x^{\prime}\right)$ the reduced density matrix of CML. The above definition in terms of an averaged quadratic form made of $\psi$ seems quite natural, especially because $\rho$ is a real symmetric, positive-definite matrix with the trace equal to 1 . The sharp brackets $\langle\ldots\rangle_{t}$ denote the time averaging

$$
\langle(\ldots)\rangle_{t}=\frac{1}{T_{s}} \sum_{t=T-T_{s}}^{T}(\ldots),
$$

where $T$ is the total simulation time and $T_{s}$ is the averaging time. In our numerical experiments $T$ has been equal to 3000 , and $T_{s}$ has been chosen to be equal to 1000 .

Let $W$ be the largest eigenvalue of $\rho$. We will say that CML is in a "condensed state" if $W$ is significantly larger than all other eigenvalues of $\rho$. If this is the case, the system possesses property (1) of the Bose-Einstein condensates.

Further, we can provide the quantitative meaning to the concept of ODLRO by saying that it is present in the system if

$$
\rho\left(x_{1}+x, x_{1}-x\right)
$$

does not go to zero with increasing $x$ [20] for any $x_{1}$. If this is the case, the system possesses the basic property (2) of the Bose-Einstein condensates.

For technical convenience, namely, to avoid dealing with too large matrices, the above definition of the reduced density matrix involves not only temporal, but also spatial averaging over $y$. Let us notice that we might equally well consider averaging over $x$ without any qualitative change in the results.

All the above definitions are modeled after the corresponding definitions in the non-relativistic classical field theory.

\section{Condensate-like features}

We have performed our numerical experiment with six values of the non-linear parameter $a(1.5+0.1 i$, $i=0,1, \ldots, 5)$, five values of the diffusion constant $d$ $(0.05 j, j=1,2, \ldots, 5)$, two different initial conditions, and periodic boundary conditions. The following initial conditions have been investigated. The first - type A - initial conditions are such that $\psi(x, y, t)$ is "excited" only at a single point at $t=0: \psi(N / 2, N / 2,0)=0.5$, and $\psi(x, y, 0)$ is equal to zero at all other $(x, y)$. By type $\mathrm{B}$ initial conditions we mean those with $\psi(x, y, 0)$ being a Gaussian function, $\psi(x, y, 0)=0.5 \exp (-0.01((x-$ $\left.\left.N / 2)^{2}+(y-N / 2)^{2}\right)\right)$. 
Tables I and II show the dependence of the largest eigenvalue of the reduced density matrix on $a$ and $d$.

TABLE I

Largest eigenvalue of the reduced density matrix. Type A initial conditions.

\begin{tabular}{c|c|c|c|c|c|c}
\hline \hline$d \backslash a$ & 1.5 & 1.6 & 1.7 & 1.8 & 1.9 & 2.0 \\
\hline 0.05 & 0.932 & 0.324 & 0.368 & 0.371 & 0.385 & 0.387 \\
0.10 & 0.933 & 0.402 & 0.375 & 0.382 & 0.389 & 0.389 \\
0.15 & 0.935 & 0.444 & 0.396 & 0.428 & 0.405 & 0.401 \\
0.20 & 0.935 & 0.390 & 0.423 & 0.436 & 0.411 & 0.405 \\
0.25 & 0.929 & 0.533 & 0.519 & 0.454 & 0.465 & 0.695
\end{tabular}

TABLE II

Largest eigenvalue of the reduced density matrix. Type B initial conditions.

\begin{tabular}{c|c|c|c|c|c|c}
\hline \hline$d \backslash a$ & 1.5 & 1.6 & 1.7 & 1.8 & 1.9 & 2.0 \\
\hline 0.05 & 0.932 & 0.333 & 0.365 & 0.368 & 0.384 & 0.386 \\
0.10 & 0.933 & 0.439 & 0.405 & 0.384 & 0.392 & 0.389 \\
0.15 & 0.934 & 0.465 & 0.658 & 0.394 & 0.403 & 0.400 \\
0.20 & 0.935 & 0.376 & 0.680 & 0.451 & 0.409 & 0.406 \\
0.25 & 0.933 & 0.404 & 0.360 & 0.420 & 0.400 & 0.397
\end{tabular}

There are several interesting observations which can be made in connection with Tables I and II. Firstly, the system exhibits one eigenvalue of the reduced density matrix which is much larger than all other eigenvalues for all values of $a$ and $d$ and both types of initial conditions. This is especially well visible for $a=1.5$, but for larger $a$ it is still true - the largest eigenvalue is at least three times bigger than the remaining ones. This is one of the most important features of the Bose-condensed matter, as explained in Sect. 2. Our system clearly has the property (2) of BEC. Secondly, unlike for the case investigated in [12], there is no trace here of the quasi-condensation, that is the presence of two or more eigenvalues close to each other. This property is perhaps somewhat astonishing because our $f(\psi)$ appears to be only a slight modification of $f(\psi)$ employed in [12].

Once the reduced density matrix $\rho$ is defined and its eigenvalues calculated, it is also possible to analyze the coherence properties of the model with the help of von Neumann's entropy. It can be defined as:

$$
S=- \text { const } \times \sum_{j} \lambda_{j} \log _{2} \lambda_{j},
$$

where $\lambda_{j}$ are the eigenvalues of $\rho$, the summation runs over all eigenvalues, and the constant prefactor is set to 1 for convenience. The minimal value of $S$ is zero - this may happen for a fully condensed system, while the maximal value appears for all $\lambda_{j}$ equal, that is, in our case, to $\log _{2} N=8$. Table III contains the values of $S$ for various $a$ and $d$ for type A initial conditions.

The von Neumann entropy measures the distance of a state of a quantum system from the pure state. In our case it can be understood as a measure of the distance from the "condensed state".

TABLE III

The von Neumann entropy as a function of parameters $a$ and $d$. Type A initial conditions.

\begin{tabular}{c|c|c|c|c|c|c|c|c|c}
\hline \hline$d \backslash a$ & 1.5 & 1.52 & 1.543 & 1.544 & 1.545 & 1.55 & 1.6 & 1.8 & 2.0 \\
\hline 0.05 & 0.786 & 0.857 & 0.910 & 2.833 & 3.517 & 3.858 & 4.806 & 4.916 & 5.167 \\
0.10 & 0.733 & 0.772 & 0.829 & 1.684 & 3.044 & 3.278 & 3.898 & 4.709 & 4.896 \\
0.15 & 0.694 & 0.738 & 0.800 & 3.079 & 2.964 & 2.809 & 3.525 & 4.072 & 4.528 \\
0.20 & 0.677 & 0.719 & 0.779 & 0.782 & 2.217 & 2.882 & 3.456 & 3.885 & 4.352 \\
0.25 & 0.637 & 0.692 & 0.864 & 2.447 & 1.457 & 2.612 & 2.860 & 3.110 & 2.129
\end{tabular}

We have had difficulties to find out regularities in the $a$ - and $d$-dependence of the maximal eigenvalue. In most (but not all) cases, the value of $W$ appears to decrease with growing $a$ for given $d$. In all cases $W$ has had the largest value for $a$ equal to 1.5 , that is below the value for which period-2 bands merge to form a single band for an individual logistic map. The picture of the dependence of $S$ on the parameters is clearer: with the notable exception of $d=0.25$ (for which the state of every cell at the time $t+1$ depends on the state of its neighbors at the time $t$, but not on the previous state of that cell itself), the entropy appears to grow monotonically with growing $c$. Also, for given $c$, one can observe that the larger diffusion constant, the smaller entropy. Of particular interest is the transitional region in the parameter space near $c=1.544$ which is very close to the point of merge of period- 2 bands. In that transitional region the entropy changes quite abruptly with $c$.

In Fig. 1 we have displayed the spatial dependence of the quantity ("one-particle correlation function") $\sigma(x)=$ $\rho(N / 2+x, N / 2-x)$ for $x=0,1,2, \ldots, N / 2-1, d=0.20$ $c=1.7$, and two types of initial conditions. 


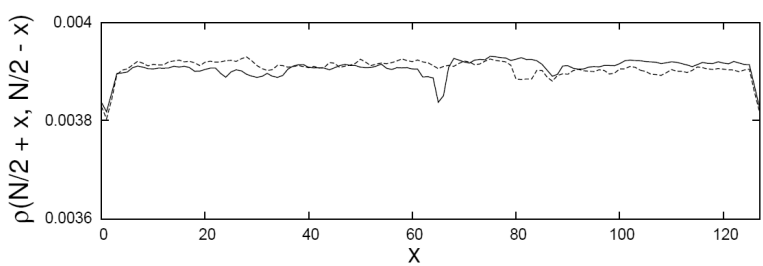

Fig. 1. Spatial dependence of the one-particle correlation functions for $d=0.20, a=1.7$; solid line: type A initial conditions, dashed line: type B boundary conditions.

While the values of the above "one-particle correlation function" for $x=0$ and $x=N / 2$ must be equal due to the boundary conditions, a strong decrease of $\sigma(x)$ for $x$ being far from 0 or $N / 2$ would have to take place if there were no long-range order. However, $\sigma(x)$ never falls below the $90 \%$ of its value for $x=0$. In fact, the change of $\sigma$ with $x$ reduces itself to very small fluctuations. We can conclude that our system exhibits the property (2) of the Bose-Einstein condensates.

One may say that the correlation length in our CML is virtually infinite, which is again a characteristic feature of the strongly condensed physical systems.

To make our case of pointing out the CML resemblance to the Bose condensates even stronger, we have checked the behavior of the field $\psi$ in momentum space. In Fig. 2a,b the plots of the moduli $|\tilde{\psi}|$ as functions of two components of their "momentum" argument are shown for periodic boundary conditions and two types of initial conditions. The function $|\tilde{\psi}(m, n)|$ is normalized in such a way that its maximal value is 1 .

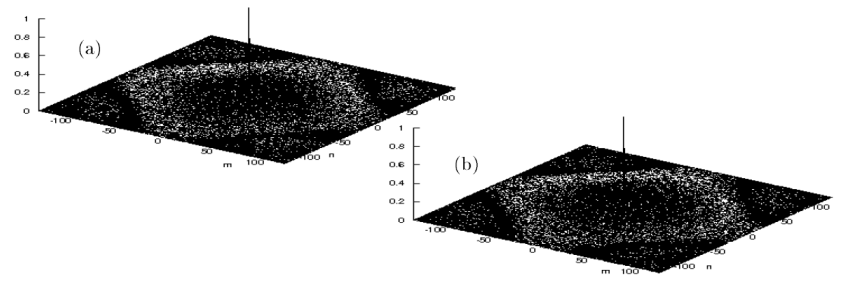

Fig. 2. The dependence of $|\tilde{\psi}|$ on the discrete vector of momentum $(m, n)$ for $d=0.20, c=3.7$, and periodic boundary conditions. The values of $|\tilde{\psi}|$ has been normalized in such a way that $|\tilde{\psi}(0,0)|=1$; (a) type A initial conditions; (b) type B initial condition.

The plots in Fig. 2 are qualitatively the same. In addition, they are representative for the entire spectrum of values of $a$ and $d$. Strong peak at the zero momentum clearly dominates all the other maxima. The fact that the zeroth mode is the only one which is so strongly populated is yet another feature of Bose-condensed system of particles - our system exhibits the property (3) of condensates.

\section{Large-scale pattern formation}

We have observed the following general rules in the process of pattern formation in our system. Firstly, the patterns are incomparably better developed (i.e. much better visible) for any "structured" initial conditions (like those considered in this work) than in the case of random initial conditions. The initial inhomogeneities (or "seeds") serve the building of large structures much better than fully random conditions, which is fairly intuitive. The patterns are best developed for smaller values of the non-linear parameter and intermediate values of the diffusion constant.

To give some examples of the pattern which emerge in two-dimensional CMLs, we show in Figs. 3 and 4 shaded-contour plots representing the values of the field $\psi(x, y)$ after 3000 time steps for periodic boundary conditions and types A and B initial conditions for several values of the parameters $a$ and $d$.

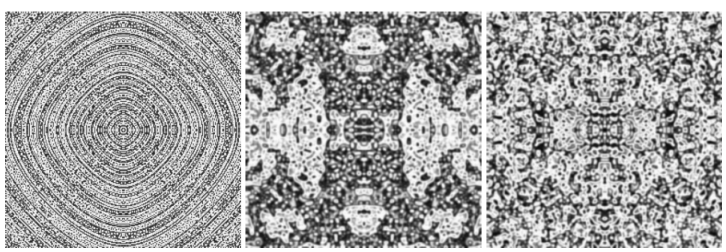

Fig. 3. Grayscale shaded contour graphics representing the values of the field $\psi$ after 3000 time steps for $d=0.05$, periodic boundary conditions, and three pairs of $a$ and $d$ values for type A initial conditions; left: $a=1.7, d=0.05$, center: $a=1.8, d=0.20$; right: $a=1.9, d=0.20$. Brighter regions are those with higher values of $\psi$.

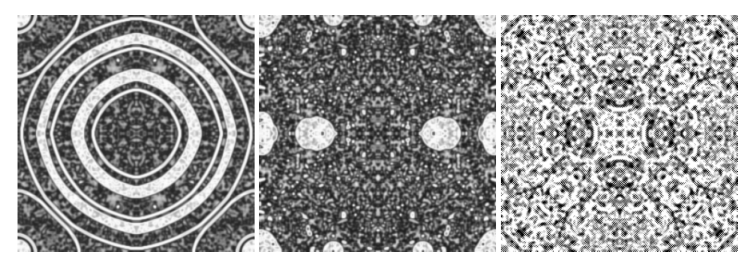

Fig. 4. Grayscale shaded contour graphics representing the values of the field $\psi$ after 3000 time steps for $d=0.05$, periodic boundary conditions, and three pairs of $a$ and $d$ values for type $\mathrm{B}$ initial conditions; left: $a=1.6, d=0.15$, center: $a=1.7, d=0.15$; right: $a=2.0, d=0.25$. Brighter regions are those with higher values of $\psi$.

Naturally, the large structures visible in Figs. 3 and 4 reflect, to some extent, the symmetry of the simulation box. It seems, however, that the essential patterns obtained in those figures are also present if the boundary conditions are set on another polygone or smooth curve (we have checked this on a triangle and an ellipse). 


\section{Concluding remarks}

Perhaps the most interesting of the various features of the considered system of coupled map lattices is that it appears to be "condensed" if the most standard measures of the classical field theory of the Bose condensates are applied. That is, for a majority of parameter values we have observed that a gap between the largest eigenvalue of the reduced density matrix and the rest has been developed.

Secondly, the prominent characteristic of the system is the presence of large-scale patterns for all values of the diffusion constant provided that the non-linear parameter is sufficiently large, that is, approximately equal to or larger than 1.5. Thirdly, a very strong dependence of both the presence and qualitative features of the patterns on the initial conditions is to be noticed.

We have, in addition, performed similar numerical experiments with the standard (Chirikov-Taylor) map, reaching practically the same conclusions, although the specific values of the dominant eigenvalue are of course different.

The critical point in any further development is, naturally, finding a physical system which could be approximated by the coupled logistic map lattices. It seems that one candidate to consider is a system of coupled lasers with periodic turning on and off of the pump. Work is in progress to provide more substance to the above remark.

\section{Acknowledgments}

It is a pleasure to thank Professors Mariusz Gajda and Marek Kuś as well as Dr. Emilia Witkowska for offering several helpful discussions. Also, we are grateful to an anonymous referee of Acta Physica Polonica A whose remarks enabled us to improve this work.

\section{References}

[1] Dynamics of Coupled Map Lattices and Related Spatially Extended Systems, Eds. J.R. Chazottes, B. Fernandez, Springer, New York 2005.

[2] A. Ilachinski, Cellular Automata. A Discrete Universe, World Sci., Singapore 2001.

[3] K. Kaneko, Prog. Theor. Phys. 72, 480 (1984).

[4] I. Waller, R. Kapral, Phys. Rev. A 30, 2047 (1984).

[5] R. Kapral, Phys. Rev. A 31, 3868 (1985).

[6] K. Kaneko, Physica D 34, 1 (1989).

[7] T. Yanagita, K. Kaneko, Physica D 82, 288 (1995).

[8] T. Yanagita, Phys. Lett. A 165, 405 (1992).

[9] P.S. Ghoshdastidar, I. Chakraborty, J. Heat Transfer 129, 1737 (2007).

[10] T. Yanagita, K. Kaneko, Phys. Rev. Lett. 78, 4297 (1997).

[11] K. Kaneko, in: Pattern Dynamics, Information Flow, and Thermodynamics of Spatiotemporal Chaos, Eds. K. Kawasaki, A. Onuki, M. Suzuki, World Sci., Singapore 1990.

[12] M. Janowicz, A. Orłowski, submitted to Phys. Rev. E.

[13] M.C. Cross, P.C. Hohenberg, Rev. Mod. Phys. 65, 851 (1993).

[14] F. Dalfovo, S. Giorgini, L.P. Pitaevskii, S. Stringari, Rev. Mod. Phys. 71, 463 (1999).

[15] A. Leggett, Rev. Mod. Phys. 73, 307 (2001).

[16] K. Góral, M. Gajda, K. Rzążewski, Opt. Express 8, 92 (2001).

[17] K. Góral, M. Gajda, K. Rzążewski, Phys. Rev. A 66 , 051602(R) (2002).

[18] K. Góral, M. Gajda, K. Rzążewski, J. Opt. B, Quantum Semiclassical Opt. 5, 96 (2003).

[19] O. Penrose, L. Onsager, Phys. Rev. 104, 576 (1956).

[20] C.N. Yang, Rev. Mod. Phys. 34, 694 (1962).

[21] D. Kadio, M. Gajda, K. Rzążewski, Phys. Rev. A 72, 013607 (2005). 